\title{
Trial Use of QOL Evaluation Management Application for Cancer Therapy
}

\author{
- Multiple Evaluation Methods and Score Analysis Methods - \\ Guen YAMADA*, Jue ZHANG ** and Takuro SAKURAI *** \\ * Graduate School of Kogakuin University, 1-24-2 Nishi-Shinjuku, Shinjuku-ku, Tokyo 163-8677, Japan \\ em19023@ns.kogakuin.ac.jp
}

\begin{abstract}
Recently, medical institutions are now trying different things to evaluate QOL more scientifically. Besides information such as cancer morbidity and mortality, a subjective outcome indicator of QOL and patient satisfaction are used to this end. However, due to the increase in the elderly population, the number of cancer patients is increasing annually. Therefore, to resolve this, it is necessary to have an evaluation that includes test tools that can evaluate an elderly cancer patient's overall condition in addition to C 30 commonly used to measure the QOL. Also, in the medical science and treatment fields, the patient's data is analyzed and used for various purposes. this paper aims to assist medical staff by managing and sampling data from multiple evaluation methods and to also provide a data analysis function. By the analysis, to make the causal relationship known and combining elements and incorporating many elements such as the patient's condition to help obtain the score evaluation time and data.
\end{abstract}

Keywords: Quality of Life, Analysis Methods, Application Development

\section{INTRODUCTION}

According to the latest cancer statistics, the probability of getting cancer in a lifetime is $62 \%$ for men and $47 \%$ for women. It is estimated that one in two people will have some form of cancer. Also, the incidence of cancer rises with age for both men and women, especially from age 50 [1]. However, thanks to the advancement of medical treatment, cancer treatment has diversified in recent years. There is now emphasis on the patient's Quality of Life (QOL) during treatment and recovery. Also, the National Comprehensive Cancer Network (NCCN) recommends the QOL evaluation for cancer treatment [2].

However, these evaluation methods are being done on paper, and changes in the format of the responses cannot be made. The response sheet has not been digitized. So it is very laborious for medical staff to use the evaluation results for research purposes. To resolve this problem, previous research tested the "QOL Kei-san App." Being a barometer of the patient's QOL, EORTC QLQ-C30 (C30) was normally used and a camera was used for data entry to see how effective it was. The trial showed that it reduced the burden on the clinical staff for entering and analyzing data [3].

However, due to the increase in the elderly population, the number of cancer patients is increasing annually. Cancer treatments are also a great burden on the patient's body, especially on the elderly. Cancer treatment policies are often not stipulated. Therefore, treatment programs are different between elderly and younger patients. To resolve this, it is necessary to have an evaluation that includes test tools that can evaluate an elderly cancer patient's overall condition in addition to C30 commonly used to measure the QOL.

Also, in the medical science and treatment fields, the patient's data is analyzed and used for various purposes. It will be used to improve patient treatment and support. In addition to the patients' electronic medical records, we expect our app to accumulate a large amount of data on patients. The evaluation methods covered in this paper are often used as a standalone to analyze and evaluate data. However, a different evaluation method may also be used to evaluate the same things. Therefore, if we can comprehensively analyze the evaluation results for different times and time periods, we can consider factoring in the evaluation method.

Therefore, this paper aims to assist medical staff by managing and sampling data from multiple evaluation methods and to also provide a data analysis function.

\section{APPLICATION DEVELOPMENT}

\subsection{Application Overview}

The prototype application was developed in iOS and Xcode, and Objective-C as the language.

The application can already be used for QOL 
evaluation such as C30. It can compute scores instantly and also manage the data. However, in recent years, medical institutions have been using other indices other than QOL evaluation. They use the index suiting the patient and conduct a subjective evaluation. A statistical analysis of the evaluation scores obtained from different evaluation methods and questionnaires is handled. Therefore, with the cooperation of cancer therapists, we had them provide 30 examples of G8 and CFS simulated data from different evaluation methods commonly used in cancer therapy for elderly cancer patients 65 or older used at the time of hospital admission and discharge (Table 1).

Table 1: Simulated data for elderly cancer patients at hospital admission and discharge

\begin{tabular}{|c|c|c|c|c|}
\hline & \multicolumn{2}{|c|}{ Admission } & \multicolumn{2}{c|}{ Discharge } \\
\hline ID & G8 & CFS & G8 & CFS \\
\hline 1 & 5 & 8 & 5 & 9 \\
\hline 2 & 10 & 8 & 11 & 8 \\
\hline 3 & 7 & 15 & 7 & 24 \\
\hline$\cdot$ & $\cdot$ & $\cdot$ & $\cdot$ & $\cdot$ \\
\hline$\cdot$ & $\cdot$ & $\cdot$ & $\cdot$ & $\cdot$ \\
\hline 30 & 5 & 19 & 5 & 14 \\
\hline
\end{tabular}

\subsection{Additional evaluation methods}

As the population ages, the age of cancer patients also increases. Currently, cancer patients who are age 65 or older account for two-thirds of such patients. This percentage is expected to increase. Therefore, among the evaluation methods provided, we added G8, CFS, and MDASI since they are frequently used methods for elderly cancer patients.

With regard to treating elderly cancer patients, the Japan Clinical Oncology Group (JCOG)'s Elderly Research Committee proposed to create two categories: "Fit: Patients who can receive the same standard treatment as healthy non-elderly patients" and "Unfit: Patients who cannot receive the same standard treatment as healthy non-elderly patients." Also, "Unfit" patients were to be further categorized into "Vulnerable: Patients who cannot receive the same standard treatment as healthy non-elderly patients, but can receive some kind of treatment" and "Frail: Patients considered to be unsuitable for aggressive treatment" [4].

G8 is a screening tool that is always used for the elderly when deciding the treatment strategy and for the evaluation of overall patient functions. It is easy and can evaluate the elderly person's physical function, cognitive function, and mental function. There are eight questions, and the G8 score can range from 0 to 17 . At medical institutions, it is difficult to decide whether the elderly patient is "frail" or "vulnerable." Therefore, the G8 tool is used to objectively evaluate the patient and decide on a treatment strategy. Also, the borderline between "frail" and "vulnerable" is determined by the G8 score. If the G8 score is $\leq 14$, the condition will be categorized as abnormal.

Also used for the elderly, CFS is a questionnaire to evaluate fatigue in cancer patients. It consists of three secondary measurements: Physical fatigue, mental fatigue, and cognitive fatigue. The maximum score is 28 for physical fatigue, 16 for mental fatigue, 16 for cognitive fatigue, and 60 for overall fatigue. The higher the score, the worse the fatigue.

MDASI comprehensively assesses the severity of cancer symptoms and the extent to which they affect daily life. It has 13 items assessing symptoms during 24 hours and 6 items assessing how they affect daily life. The score is obtained by averaging the score of all the items for the symptom severity and how they affect daily life.

\subsection{Implementation of analysis function}

In medical research, selecting the statistical method suited for the data is important at medical institutions. The simulated data in this study consists of the G8 and CFS evaluation scores of an elderly patient. It is bivariate data divided for the hospital admission time and the discharge time (30 observation samples). The bivariate amount for the changes at the hospital admission time and the factors for the grouping and connection between the two groups can be studied with the application's analysis function to test the difference of the averages between the two groups. Also, the G8 score is used to categorize patients into the "frail" or "vulnerable" group. After the G8 score data is entered, the application has a categorization function (Fig. 1) to recognize the G8 scores and automatically categorize them into groups based on a score of G8 $\leq 14$ or G8 $\geq 15$.

\begin{tabular}{ccccccc|}
\hline & $<=14$ & & \multicolumn{3}{c|}{$>=15$} \\
ID & 68 & CFS & D & G8 & CFS \\
1 & 5 & 9 & 6 & 15 & 28 \\
2 & 11 & 8 & 18 & 16 & 9 \\
3 & 7 & 24 & 21 & 15 & 44 \\
\hline
\end{tabular}

Fig 1: Categorization function by G8 score.

Regarding the function for examining the difference of the averages between the two groups, we have to keep in mind that the method of calculating the tested statistics will differ slightly. The t-test must be selected to suit one of two major conditions: Whether the sample data to be 
tested is geared for the two groups or whether it can be divided equally. Therefore, for the test, this function provides an option to select the data's suitability. If both groups are not suitable, an equal division test (F-test) will be conducted first. If equal division is deemed to be feasible, the Student's t-test will be conducted. If equal division is not feasible, then a Welch's t-test is to be conducted. This was the basic stance taken (Table 2).

Table 2: t-test applied according to pattern

\begin{tabular}{|c|c|}
\hline Pattern & t-test applied \\
\hline Corresponds to data & Applicable t-test \\
\hline $\begin{array}{c}\text { No correspondence to } \\
\text { data, and equal division can } \\
\text { be assumed }\end{array}$ & Student's t-test \\
\hline $\begin{array}{c}\text { No correspondence to } \\
\text { data, and equal division can } \\
\text { not be assumed }\end{array}$ & Welch's t-test \\
\hline
\end{tabular}

In accordance with the patterns shown in Table 2, the actual applicable t-test, Student's t-test, Welch's t-test, and F-test were implemented. In addition to the above, classes and methods were created to obtain the basic statistics (arithmetic mean, variance, standard deviation), correlation function, t-value, $\mathrm{F}$ value, $\mathrm{p}$-value, etc. Also, for the test's judging standard, there are two methods: 1 . Comparison of the test statistic t-value and the borderline value. 2. Comparison of the test statistic $p$-value and the significance standard. Since the former requires a borderline value, a t-distribution table was created by manipulating the database file (SQLite) using Objective-C. Regarding the significance standard, $\alpha=$ 0.05 is normally often taken, but there are cases where $\alpha=$ 0.01 is set strictly. Therefore, both have been implemented in the application. By using the above statistical classes, we succeeded in obtaining the statistical values in the program. Also, we actually fed the simulated data and designed it so that the statistics amount matching the test method would be output to the screen.

\section{VERIFICATION EXPERIMENT}

\subsection{Overview of experiment}

From the simulated data, to study the connection between the G8 score and CFS score for the elderly, the application's test function was used to conduct a verification experiment.

Since fatigue is felt differently by different people, it is regarded to be difficult to measure. Therefore, we checked for any difference in the CFS and G8 scores at the time of hospital admission and discharge. CFS mainly evaluates the cancer patient's fatigue, and G8 evaluates the cancer patient's mental aspects. Also, CFS is a questionnaire that evaluates the patient's fatigue at the current time. And G8 is a questionnaire for evaluation applicable to a fixed period of time. We compared the current fatigue condition and the fatigue during a fixed period of time and tried to find a connection.

\subsection{Experiment procedore}

We started up the iOS simulator attached to Xcode and in the application, selected the t-test suited for the pattern in accordance with Table 2. We decided which test function to use. We then entered the 30 examples of simulated data on the data entry screen, and we output all the test statistics to the results screen (Figs. 2 and 3).

With the simulated data, we could use the G8 scores to categorize patients as "frail" or "vulnerable." Therefore, we split them into the time of hospital admission and discharge. For the "frail" CFS (G8 $\leqq 14$ patients) and "vulnerable" CFS (G8 $\geqq 15$ patients), we tested the difference between the two groups not corresponding to the time of hospital admission and discharge. Also, since this data measures the value for the period of hospital admission to the discharge for each ID, we can assume there is a corresponding connection, so we tested the difference between the two groups corresponding to G8 and CFS. Also, when the former was implemented, we used the implemented separation function (Fig. 1) to automatically separate them into groups for relative comparison. We then tested the bivariate. Also, in this experiment, a significance standard of $\alpha=0.05$ was used, and a total of four patterns were implemented in the test for both sides.

\subsection{Analysis results and considerations}

Fig. 2 shows the results of examining the difference between the two non-corresponding groups for the "frail" CFS score (G8 $\leqq 14$ patients) and "vulnerable" CFS score (G8 $\geqq 15$ patients) at the time of hospital admission and discharge. For the two non-corresponding groups, we checked for any difference in the CFS score indicating fatigue. For the "frail" condition and "vulnerable" condition, we looked for any difference in the fatigue-indicating CFS score between the two non-corresponding groups. We found that at the time of hospital admission, it was " $\mathrm{t}=0.38, \mathrm{df}=6, \mathrm{p}=.717 \mathrm{n}$ and at the time of discharge, $" t=0.33, d f=28, p=.743 "$. Since it was $\mathrm{p}<.05$ for both, no significant difference was recognized. Also, the F-test was conducted before the t-test of the two non-corresponding groups. The results showed "F=3.19, df=23, 5, p=.024" for the time of hospital admission and "F=2.15、 $\mathrm{df}=22 、 6 、 \mathrm{p}=.087$ " for 
the discharge time. For the hospital admission, the Welch's t-test (Fig. 2 left) was used. For the discharge, the Student's t-test (Fig. 2 right) was used to output the test results.

Fig. 3 shows the results of examining the difference between the two groups corresponding to the G8 score and the CFS score that evaluated the different times of their hospital admission and discharge. Regarding the scores obtained at the time of hospital admission and discharge, we looked for any difference between the two corresponding groups. We found G8 to be $" t=-0.095$, $\mathrm{df}=29, \mathrm{p}=.924 "$ and CFS to be "t=-0.359, $\mathrm{df}=29, \mathrm{p}=.722 "$. Since both were $\mathrm{p}<.05$, a significant difference was not recognized.

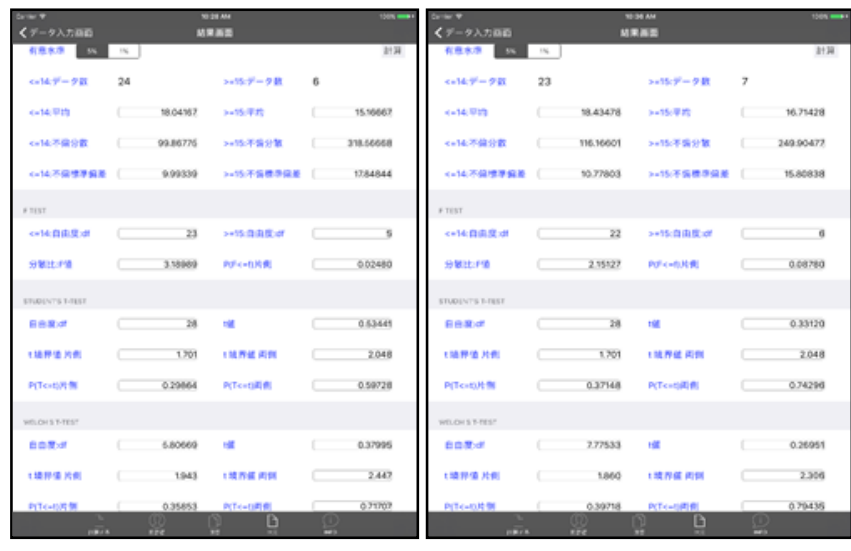

Fig 2: Test results of the two corresponding groups

(Left Fig. is admission, and right Fig. is discharge)

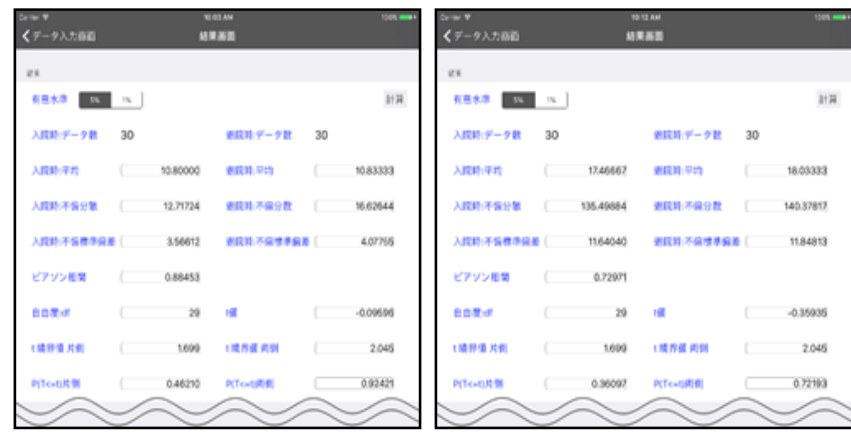

Fig 3: Test results of the two corresponding groups (Left Fig. is G8, and right Fig. is CFS)

The respective patterns did not show any statistical significance for any period or group. However, they suggest that elderly patients were able to maintain their condition at the time of hospital admission and discharge after a period of treatment such as therapy or surgery. However, when examining patient data individually and comparing the change in their scores for their hospital admission and discharge, many patients did not show improvement. But still, there were patients who did greatly improve. This may be due to a wide variety of physical, mental, and environmental factors such as the cancer stage, symptoms, and basic information.

\section{CONCLISIONS AND FUTURE CONSIDERATIONS}

This paper focuses on the problems of the elderly. It has been added to the QOL application that developed an evaluation method commonly used for the elderly. The implementation has been successful, and it is also possible to add other evaluation methods for cancer patients henceforth. Also, to focus on the elderly patient's condition, a function has been added to categorize the condition in accordance with the patient's G8 score. We considered having an analysis function that can study the differences in the evaluation scores. The result: By automatically categorizing the elderly patient's condition and setting the evaluation score, we succeeded in implementing the t-test that studies the difference between the two groups. Since every patient is full of diversity, it is probably not easy to obtain detailed relevance of the evaluation score just by using the evaluation score from the test result.

Things to do next: To study the data affecting the patient. By doing so, to make the causal relationship known and combining elements and incorporating many elements such as the patient's condition to help obtain the score evaluation time and data.

\section{REFFERENCES}

[1] http://ganjoho.jp/public/

[2] NCCN http://www.nccn.org/

[3] Yukiya Hunada; "Study of QOL Assessment Management Application” , 2017

[4] Kenichi Nibu; "Cancer treatment for the elderly" , 2018 